\title{
Dynamics Modeling and Simulation Analysis for Wood High-speed Motorized Spindle
}

\author{
Wei Zhang ${ }^{1,2}$, Shuangyong Wang ${ }^{1}$, Yang Zhang ${ }^{3}$, Hailong $\mathrm{He}^{1}$ and Pengfei \\ Zhang $^{1}$ \\ ${ }^{1}$ Beijing Forestry Machinery Research Institute of the State Forestry \\ Administration, Beijing, 100029, China \\ ${ }^{2}$ Forestry New Technology Research Institute, Chinese Academy of Forestry, \\ Beijing, 100091, China \\ ${ }^{3}$ Beihang University, Beijng, 100191, China \\ zwei611@hotmail.com
}

\begin{abstract}
In this paper, considering the main factor in the high-speed processing, including the mass of the spindle, the offset of the centroid, bearing span, bearing stiffness and spindle speed, a dynamic model of the spindle-bearing system is established. After carrying out a simulation by Matlab, vibration curve of the spindle-bearing system is obtained, and influence on the vibration characteristics of the spindle bearing system by the centroid offset is analyzed. These works provide the basis for further studies on the vibration mechanism of the high-speed motorized spindle.
\end{abstract}

Keywords: Wood machining, High-speed spindle, Dynamic model, Simulation

\section{Introduction}

With the wood processing technology developing towards higher speed and precision and intelligence, the high speed electric spindle technology for woodworking is becoming the core component of the wood working machinery [1] and its performance becoming a key role of the machinery. Due to the natural construct and the anisotropy of the wood, the unbalance caused by the scab of the wood will lead to vibration of the electric spindle [2-3] in high speed machinery. Besides, the offset error of the mass center of the electric spindle and torsion deformation also result in vibration of the electric spindle. The vibration will increase the machining error of the cutter, especially in high speed machining, which will seriously influence the machining precision of the machinery [45]. What's more, if the vibration is too strong, it may damage the mechanical structure of the machining devices. So it is necessary to deeply research the mechanistic of the high speed electric spindle vibration, and to analyze the factors that affect the vibration.

In the research of high-speed motorized spindle characteristics, Chen Xiaoan conducts dynamic analysis in the method of finite element, establishing high-speed motorized spindle dynamic model and generalizing unbalanced expression of high-speed motorized spindle in various offset states [6]. Chen and Hwang analyze factors that lead to stiffness decreasing and influence of dynamic features [7]. Meng Jie simplifies segmentation model of spindle with transition matrix method, and obtains first order vibration mode and spindle natural frequency with the method of finite element, which is basically consistent with the calculating result of transition matrix [8]. Wu Shaolong obtains every frequency order and vibration mode of the spindle in mode analysis of motorized spindle, and finds out frequency requirements of anti-vibration and the influence of front bearing stiffness and damp on the spindle [9]. Xu Xin establishes low-wear magnetic suspension motorized spindle experiment equipment through bringing in $\mathrm{AMB}$ and $\mathrm{PMB}$, and analyzes its dynamic performances [10]. In this paper, simplified spindle-bearing 
dynamic model is established focusing on centroid offset in the spindle production and setting up, and dynamic characteristics and vibration mechanism of spindle is further analyzed.

\section{Dynamic Modeling}

In the dynamic research of the wood high-speed motorized spindle, wood features and spindle structure should be considered including wood texture, scars and cutting force, which lead to unbalance and vibration of the spindle. Meanwhile, relative parts of the spindle should be emphatically considered including rotor, stator and bearing, as well as setup error, clearance between stator and rotor, bearing stiffness, lubrication and cooling. To simplify, only several main factors - mass of the spindle, the offset of the mass center, bearing span, bearing stiffness and spindle speed - are taken into consideration. To start with, the dynamic model of the spindle-bearing system is established. From the dynamical differential equation and the initial conditions of the system, the movements of the spindle can be computed, and then the vibration characteristics can be analyzed.

Most previous studies of dynamic analyses simplified bearing as rigid connection, which is allowed only when deformation of the bearing is small. But for the woodworking high speed electric spindle, the bearings should be considered as elastic connection [11]. Hence, in this paper, each bearing on the spindle is simplified as a pair of springs in the horizontal and vertical direction, and the stiffness of the spring is equal to the stiffness of the bearing, noted as $k_{1}$ and $k_{2}$.
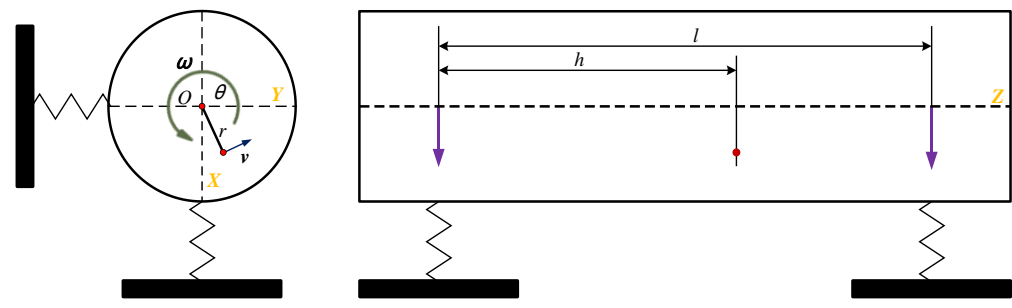

Figure 1. The Simplified Model of the Spindle-Bearing

The simplified model of the spindle-bearing is shown in Figure 1. The origin of the reference frame is defined as the intersection of the spindle rotation axis and the center plane of the left bearing, noted as $O$. Z -axis is coincide with the rotation axis of the spindle and pointed from the left bearing to the right bearing. $X$-axis is vertical to the $Z$ axis and points downward. $Y$-axis, together with $X$-axis and $Z$-axis, constitutes a righthanded Cartesian coordinate system. Assume that the mass of the spindle is $m$, the distance of two bearing is $l$, and the rotation angle of the spindle is $\theta$. In the initial condition, the coordinates of mass center of the spindle is $P_{m}=(r, 0, h)$, where $r$ is the offset of the mass center from the rotation axis, and $h$ is the length from the mass center to the left bearing. The rotation angle $\theta=0$ at the beginning.

If the angular speed of the spindle remains constant, noted as $\omega$, then for time $t$, we have:

$$
\theta=\omega t
$$

Then the coordinates of the spindle mass center are:

$$
P_{m}=(r \cos \omega t, r \sin \omega t, h)
$$

The centripetal accelerate of spindle rotation is:

$$
\boldsymbol{a}_{\boldsymbol{n}}=\left[\begin{array}{c}
\omega^{2} r \cos \omega t \\
\omega^{2} r \sin \omega t \\
0
\end{array}\right]
$$


This centripetal acceleration is caused by the elastic force of the bearings. Examine the acceleration distribution of the spindle in $\mathrm{XZ}$ plane, which is shown in Figure 2.
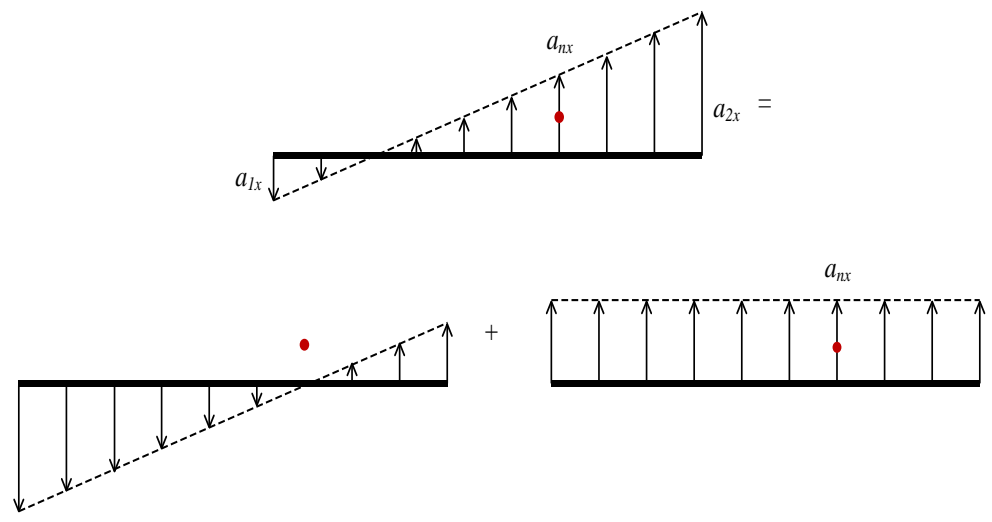

Figure 2. Acceleration Distribution of the Spindle in XZ Plane

Here $a_{n x}=\omega^{2} r \cos \omega t$. Decompose the acceleration into two part: rotation acceleration that around the mass center in $\mathrm{XZ}$ plane, and the translation acceleration of the spindle. As the rotation acceleration is 0 at the mass center, the translation acceleration is equal to the centripetal acceleration. Assume that the angular acceleration in $Y$-axis is $\beta_{Y}$, the deformation of two bearings in $\mathrm{XZ}$ plane are $\varepsilon_{1 x}$ and $\varepsilon_{2 x}$, the acceleration of the spindle at two bearings in XZ plane are $a_{1 x}$ and $a_{2 x}$, and the moment of inertia about $Y$-axis is $I_{Y}$, then:

$$
\frac{a_{1 x}-a_{n x}}{h}=-\frac{a_{2 x}-a_{n x}}{l-h}=\beta_{Y}
$$

And

$$
\left\{\begin{array}{l}
F_{1 x}=k_{1} \varepsilon_{1 x} \\
F_{2 x}=k_{2} \varepsilon_{2 x} \\
F_{1 x} h-F_{2 x}(l-h)=\beta_{Y} I_{Y} \\
F_{1 x}+F_{2 x}=m a_{n x} \\
\frac{a_{1 x}-a_{n x}}{h}=\beta_{Y} \\
\quad-\frac{a_{2 x}-a_{n x}}{l-h}=\beta_{Y} \\
\ddot{\varepsilon}_{1 x}=a_{1 x} \\
\ddot{\varepsilon}_{2 x}=a_{2 x}
\end{array}\right.
$$

In this equation, the unknown variables are: $a_{1 x}, a_{2 x}, \varepsilon_{1 x}, \varepsilon_{2 x}, F_{1 x}, F_{2 x}, \beta_{Y}$. Solve the equation and we get:

$$
\begin{gathered}
\ddot{\varepsilon}_{1 x}=\frac{1}{I_{Y}}\left(k_{1} \varepsilon_{1 x} l h-m a_{n x}(l-h) h\right)+a_{n x} \\
\ddot{\varepsilon}_{2 x}=\frac{1}{I_{Y}}\left(k_{2} \varepsilon_{2 x} l(l-h)-m a_{n x} h(l-h)\right)+a_{n x}
\end{gathered}
$$

These are second-order differential equations. If the coefficients in the right side are constant, it is easy to get the analytical solutions. Considering that $a_{n x}=\omega^{2} r \cos \omega t$ is time-varying, substitute it into the equations and we obtain:

$$
\ddot{\varepsilon}_{1 x}=\frac{1}{I_{Y}}\left(k_{1} \varepsilon_{1 x} l h-m(l-h) h \omega^{2} r \cos \omega t\right)+\omega^{2} r \cos \omega t
$$




$$
\ddot{\varepsilon}_{2 x}=\frac{1}{I_{Y}}\left(k_{2} \varepsilon_{2 x} l(l-h)-m h(l-h) \omega^{2} r \cos \omega t\right)+\omega^{2} r \cos \omega t
$$

These are the dynamic differential equations in $\mathrm{XZ}$ plane. In $\mathrm{YZ}$ plane, the form of the equation is similar:

$$
\begin{gathered}
\ddot{\varepsilon}_{1 y}=\frac{1}{I_{X}}\left(k_{1} \varepsilon_{1 y} l h-m(l-h) h \omega^{2} r \sin \omega t\right)+\omega^{2} r \sin \omega t \\
\ddot{\varepsilon}_{2 y}=\frac{1}{I_{X}}\left(k_{2} \varepsilon_{2 y} l(l-h)-m h(l-h) \omega^{2} r \sin \omega t\right)+\omega^{2} r \sin \omega t
\end{gathered}
$$

Where $\varepsilon_{1 y}$ and $\varepsilon_{2 y}$ are the deformation of two bearings in YZ plane, $I_{X}$ is the moment of inertia about $X$-axis.

The analytical solutions of the equations are very complicated, so numerical simulation method is used to compute and analyze the vibration modes of the spindle at two bearing.

\section{Simulation and Analysis}

The process of the simulation is shown in Figure 3, and the simulation is conducted with the Matlab.

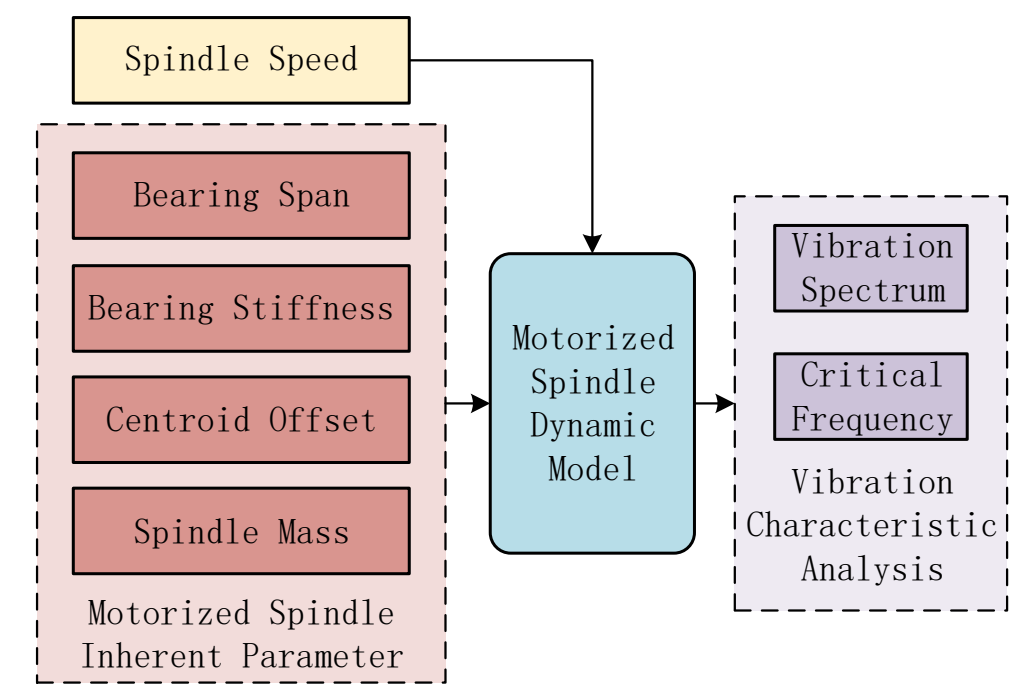

Figure 3. Flow Chart of the Motorized Spindle Dynamic Model Simulation

The simulation parameters: spindle mass is $\mathrm{m}=2 \mathrm{~kg}$; the stiffness of the left and the right bearing is $\mathrm{k}_{1}=5 \times 10^{8} \mathrm{~N} / \mathrm{m}$ and $\mathrm{k}_{2}=6 \times 10^{8} \mathrm{~N} / \mathrm{m}$, respectively; the bearing span is $1=0.2 \mathrm{~m}$, the distance from the centroid of the spindle to the left bearing is $\mathrm{h}=0.08 \mathrm{~m}$; the centroid offset from the spindle axis $\mathrm{r}=2 \times 10^{-4} \mathrm{~m}$, the rotary inertia of the spindle around the $\mathrm{X}$-axis and the $\mathrm{Y}$-axis is $\mathrm{I}_{\mathrm{X}}=\mathrm{I}_{\mathrm{Y}}=m l^{2} / 18$; the spindle speed is $\mathrm{n}=20000 \mathrm{r} / \mathrm{min}$, and the corresponding angular velocity is $\omega=2 \pi \mathrm{n} / 60$.

The initial condition of the four differential equation shown above are set as: $\varepsilon(0)=0, \dot{\varepsilon}(0)=0$. With numerical integration, the displacement of the spindle at each bearing can be obtained. The simulation result are shown in Figure 4 and Figure 5. 


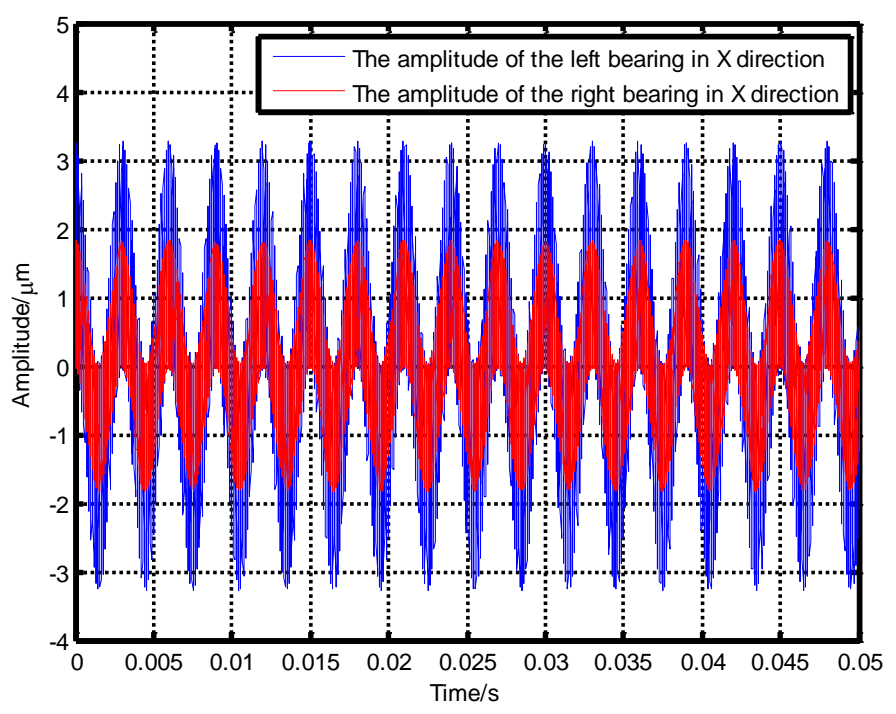

Figure 4. The Vibration Curve of the Spindle at Bearing in X Direction

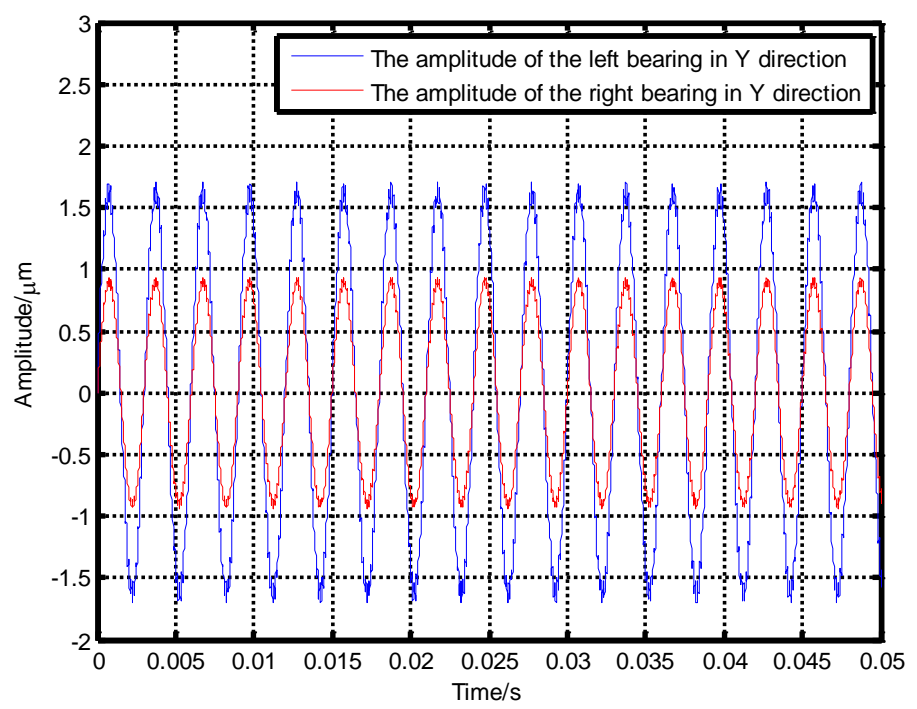

Figure 5. The Vibration Curve of the Spindle at Bearing in Y Direction

It can be seen in the time domain curve that, driven by the external cycle force, the spindle at both bearing presents typical cycle vibration, and the vibration amplitude at left bearing is larger than the right in both $\mathrm{X}$ and $\mathrm{Y}$ direction. Meanwhile, influenced by the elastic of the bearing, the spindle has a higher frequency vibration mode. For the convenience we take a spectrum analysis of the vibration. The frequency spectrum is shown in Figure 6 and Figure 7. 


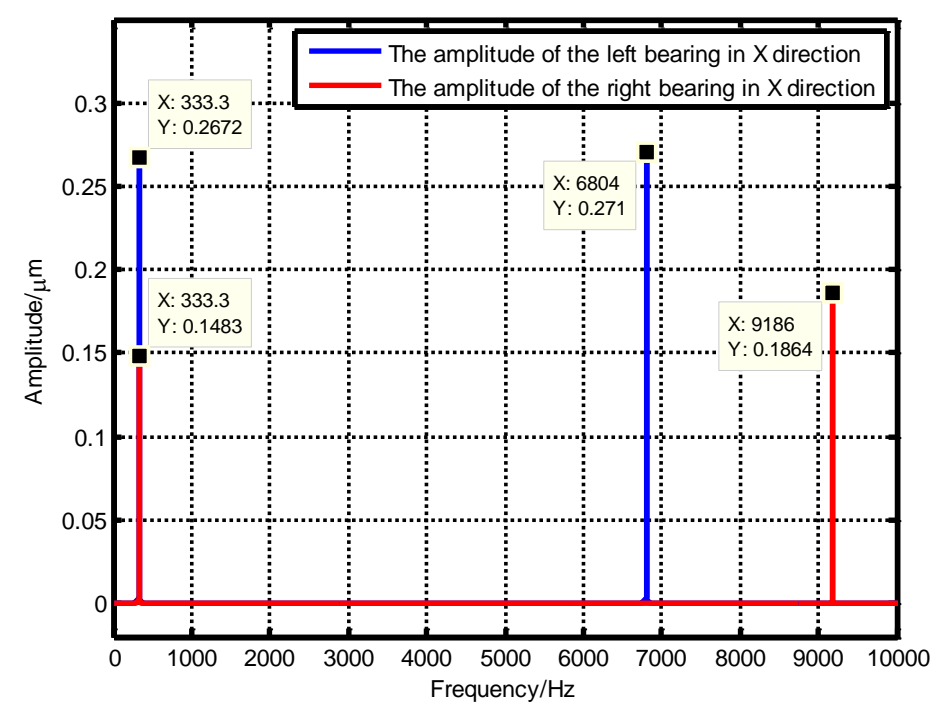

Figure 6. The Frequency Spectrum of X Direction

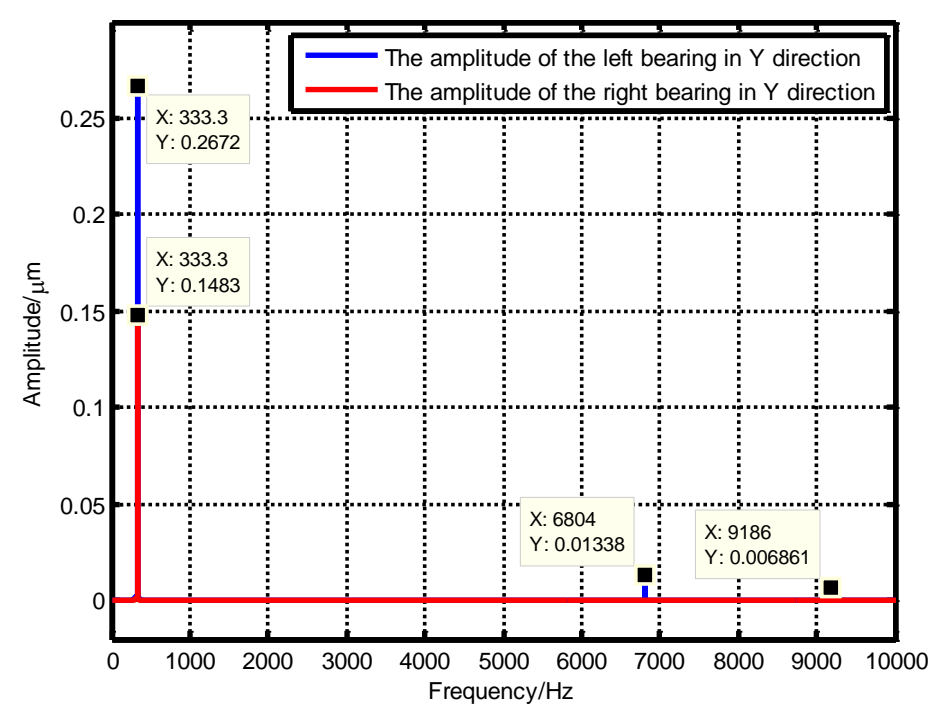

Figure 7. The Frequency Spectrum of Y Direction

From these frequency spectrum, the vibration is a superposition of two mode. The frequency of the lower mode is about $333 \mathrm{~Hz}$. The rotation speed of the spindle is $\omega=$ $2 \pi \times 20000 / 60=2.0944 \times 10^{3} \mathrm{rad} / \mathrm{s}$, while $\omega=2 \pi f$, so $f=\omega / 2 \pi=333.33 \mathrm{~Hz}$, which is in agreement with the simulation.

The higher mode is caused by the elastic of the bearing, and different stiffness of the bearing can result in a different frequency. As shown in Figure 6 and Figure 7, the higher vibration frequency at left bearing is $6804 \mathrm{~Hz}$ and the right is $9186 \mathrm{~Hz}$.

In the analysis above, the simulation condition is constant. However, the motorized spindle centroid offset fluctuates due to rotation speed, temperature, cutting force and the bearing wear, so it is necessary to simulate and analyze the spindle vibration with different centroid offset. In the simulation the centroid offset changed with other things equal, the influence of the centroid offset on the spindle vibration can be obtained, shown in Figure 8-11. 


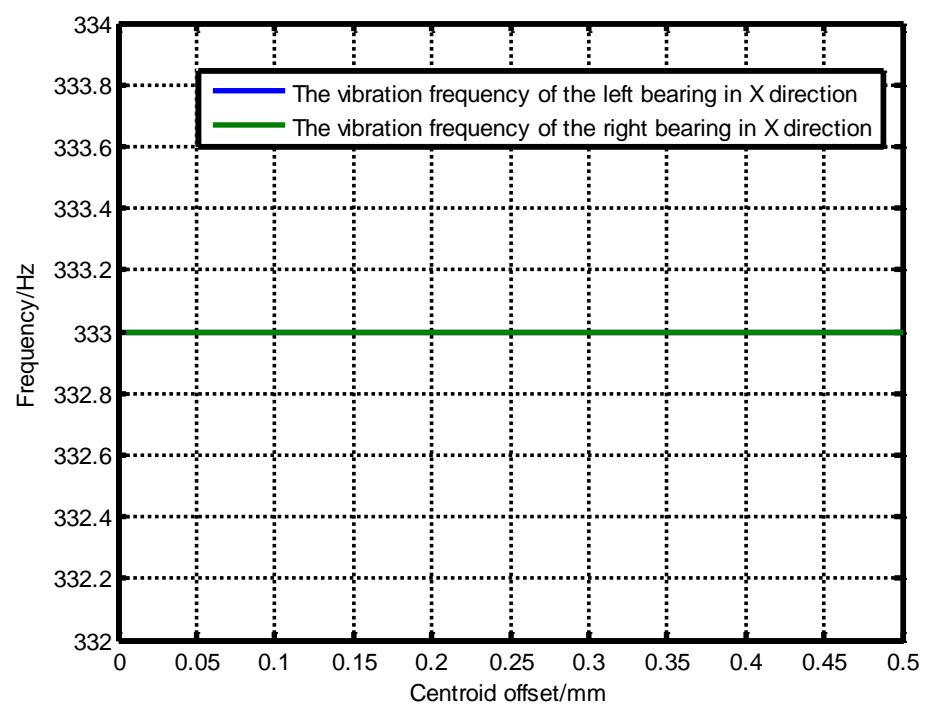

Figure 8. Influence of the Centroid Offset on the Low Frequency Vibration Frequency

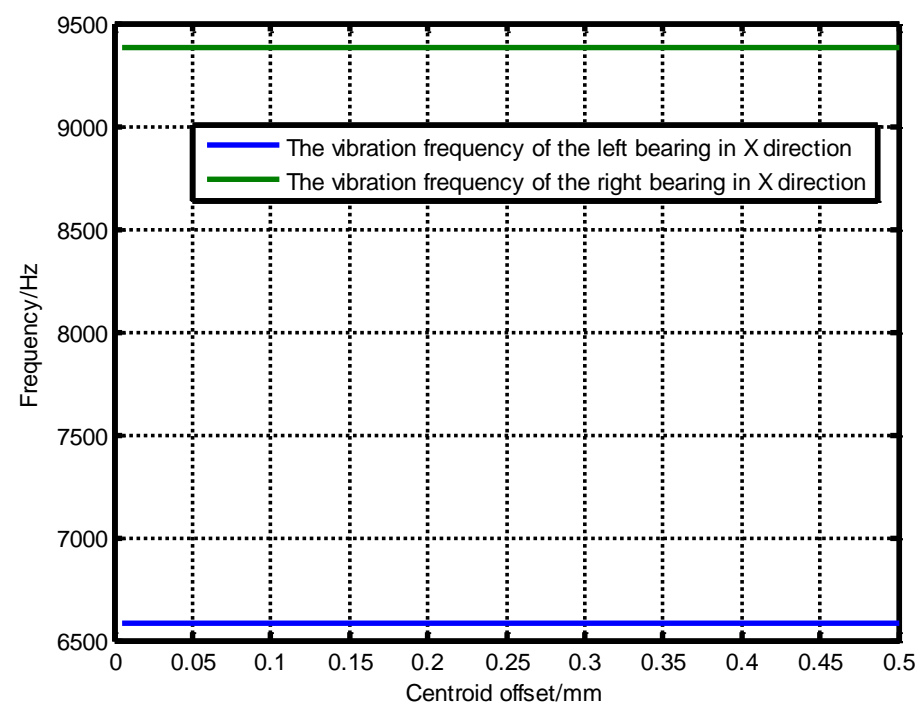

Figure 9. Influence of the Centroid Offset on the High Frequency Vibration Frequency 


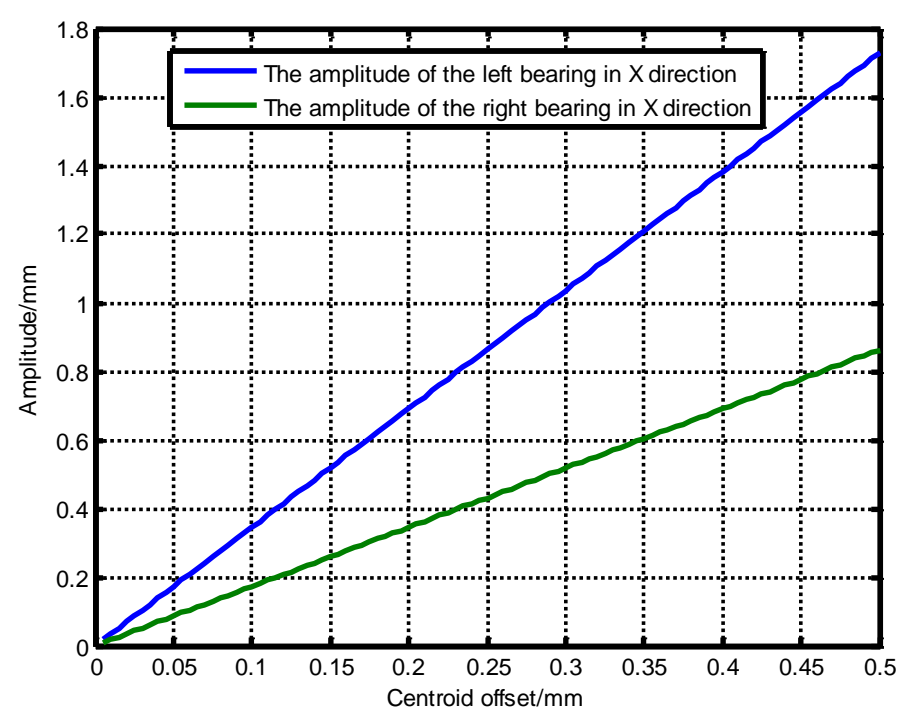

Figure 10. Influence of the Centroid Offset on the Low Frequency Vibration Amplitude

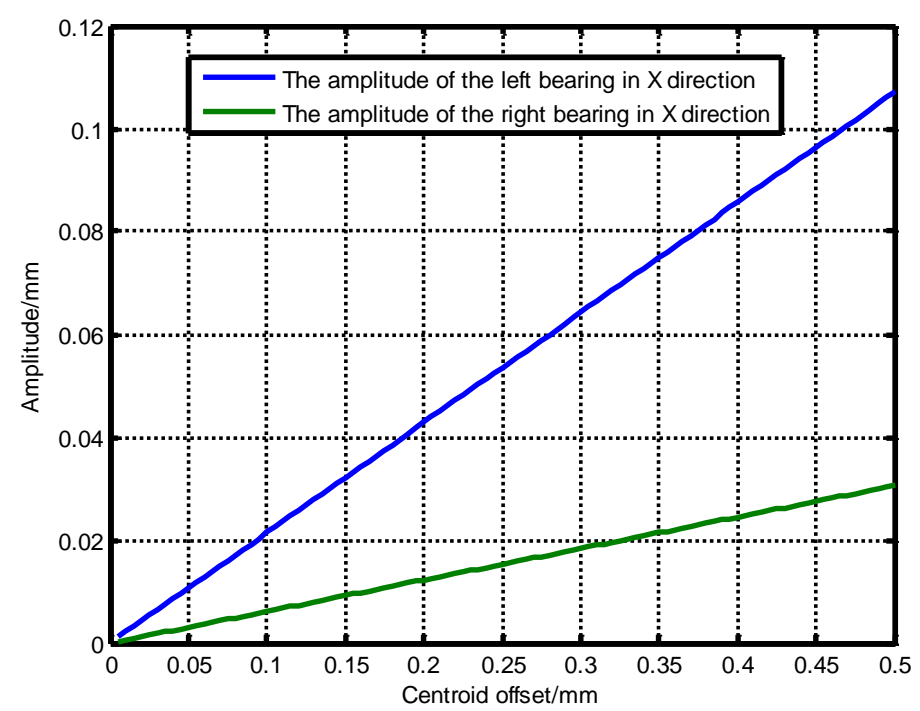

Figure 11. Influence of the Centroid Offset on the High Frequency Vibration Amplitude

It can be seen that, centroid offset hardly influence the frequency of both vibration mode, but the amplitude of two vibration mode increases along with the centroid offset. Comparing the vibration at left and the right bearing, the amplitude of the vibration at left bearing is over twice than the right, which is constant with the factor that the centroid of the spindle is closer to the left bearing.

\section{Conclusion}

In this paper, with several main factors on the wood high-speed motorized spindle in actual working conditions being considered, the spindle-bearing dynamic model is established and the influence of the centroid offset on the spindle vibration is analyzed. Through the theoretical derivation and numerical calculation, it is concluded that the 
frequency of the spindle vibration is free from the centroid offset, but the amplitude has a linear relationship with the centroid offset. So the centroid offset is not responsible for the accidental increase of the spindle vibration frequency but for the amplitude increase. This provides a new suggestion for further analyzing vibration mechanism of the highspeed motorized spindle.

\title{
Acknowledgements
}

This work was financially supported by the Agricultural Science and Technology Achievements Transformation Fund Project of MOST (2013GB24320617).

\section{References}

[1] W. Zhang, "Present situation of domestic and International high speed electric spindle technology for wood processing", Woodworking Machinery, no. 1, (2012).

[2] Z. H. Cui and W. Zhang, "The utilization of high speed spindle in wood processing", Wood Processing Machinery, no. 6, (2008).

[3] D. G. Ma, X. B. Jiang and Y. Ma, "Design and analysis of dynamic characteristics of motorized spindle for high speed wood-working machinery", Wood Processing Machinery, no. 3, (2012).

[4] J. He, Q. H. Zhang, G. X. Sun, J. C. Yang and J. B. Xiong, "A Vibration Signal Analysis Method based on Enforced De-Noising and Modified EMD”, International Journal of Signal Processing, Image Processing and Pattern Recognition, vol. 8, no. 1, (2015).

[5] T. Eguchi and T. Nakamiya, "An Improved Component-Mode Synthesis Method to Predict Vibration of Rotating Spindles and Its Application to Position Errors of Hard Disk Drives", Journal of Vibration and Acoustics, vol. 128, (2006).

[6] X. A. Chen, W. Q. Chen, H. M. Kang and Y. He, "Dynamic analysis of high speed motorized spindles with eccentrics", Journal of Chongqing University, vol. 35, no. 3, (2012).

[7] J.-S. Chen and Y.-W. Hwang, "Centrifugal force induced dynamics of a motorized high-speed spindle", The International Journal of Advanced Manufacturing Technology, no. 30, (2006).

[8] J. Meng and X. A. Chen, "Transference matrix method for dynamics analysis of motorized spindle", Journal of Machine Design, vol. 25, no. 7, (2008).

[9] S. L. Wu and Y. X. Zhang, "Dynamics analysis of high-speed spindle based on ANSYSWorkbench", Modular Machine Tool \& Automatic Manufacturing Technique, no. 9, (2010).

[10] X. Xu, Z. Y. Xie and Y. W. Long, "Dynamic characteristics of low loss motorized spindle suspended by magnetic bearings", Journal of Aerospace Power, vol. 29, no. 2, (2014).

[11] Y. Z. Cao and Y. Altintas, "A General Method for the Modeling of Spindle-Bearing Systems", Journal of Mechanical Design, vol. 126, (2004).

\begin{abstract}
Author
Zhang Wei, Ph.D., chief expert, professor researcher, director of forestry machinery branch, Beijing Forestry Machinery Research Inst. of The State Forestry Administration, mainly engages in forestry equipment design theory and methods. Bachelor's degree in Forestry Machinery and Master's degree in Forestry and Woodworking Machinery had been received in Northeast Forestry University, and $\mathrm{PhD}$ in Wood Science and Technology had been received in Chinese Academy of Forestry. Deputy secretary general of China Forestry Machinery Association Panel Machinery Branch, forestry machinery branch director of Chinese Society of Forestry, member of the National Forest Fire Standardization Committee.
\end{abstract}

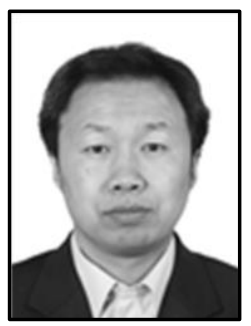


International Journal of Signal Processing, Image Processing and Pattern Recognition Vol.9, No.1 (2016) 